(REVIEW ARTICLE)

\title{
Current aspects in the management of COVID 19
}

\author{
Shashidhar Binnamangala Gangappa ${ }^{1}$, Abhishekh Basavarajegowda ${ }^{2,}{ }^{*}$ and Srikanth Umakanthan ${ }^{3}$ \\ ${ }^{1}$ Consultant, Critical Care, Vikram Hospital, Bengaluru, Karnataka, India. \\ ${ }^{2}$ Associate Professor, Department of Transfusion Medicine, Jawaharlal Institute of Postgraduate Medical Education and \\ Research(JIPMER), Puducherry, India \\ ${ }^{3}$ Faculty, Department of Paraclinical Sciences, The University of the West Indies, Mount Hope, Trinidad and Tobago
}

Publication history: Received on 29 September 2020; revised on 07 October 2020; accepted on 11 October 2020

Article DOI: https://doi.org/10.30574/wjarr.2020.8.1.0363

\begin{abstract}
Diagnosis and management of emerging infections like COVID 19 is a combination of clinical, epidemiological, and laboratory considerations. The mainstay of diagnosis is RT-PCR from oro-nasal swabs. Other blood investigations for hematology and biochemistry and imaging for chest infections can be supportive and may have prognostic value. Clinical management mainly involves supportive care as any acute care lung infections and sepsis, oxygen therapy, either invasive or non-invasive, as applicable. Corticosteroid therapy and anticoagulation are known to somewhat effective. Ivermectin, Tocilizumab, Favipiravir, and Remdesivir have shown to be of some benefit. Convalescent plasma therapy has been tried with not much evidence of benefit. Conclusive evidence for any particular modality of treatment is yet to be published. It is pertinent to understand that the successful management of critically ill patients with multisystem involvement requires a concerted holistic approach with multidisciplinary care.
\end{abstract}

Keywords: Covid-19; Management; Diagnosis; Ventilation

\begin{abstract}
Abbreviations
HFNC - High flow nasal cannula, ARDS NET- Acute Respiratory distress syndrome network. HCW- Health care workers, RTPCR-Reverse Transcriptase Polymerase Chain Reaction.
\end{abstract}

\section{Introduction}

COVID 19, which first emerged in China in late December 2019, spread pandemically to various parts of the world. The first case in India was reported in late January 2020. For an accurate diagnosis of emerging infections like COVID 19, a combination of clinical, epidemiological, and laboratory considerations are essential. The choice of the test and interpretation depends heavily on disease activity and community prevalence. The management currently is mostly symptomatic, albeit a few specific drugs which have started showing some benefit [1].

\section{Diagnosis}

The current laboratory standard of diagnosing the acute phase of COVID 19 during the pandemic is by RT-PCR, as it is the most sensitive and specific test. RT-PCR with swabbing samples from naso- or oro-pharynx will remain the standard for diagnosis of COVID 19 during the acute phase. In cases where the suspicion of COVID 19 remains high with the RT PCR from nasal swab being negative, testing may be repeated from a sample from the upper airway or from the lower respiratory tract in patients who are intubated with in-line suction [2]. The various testing platforms available for

\footnotetext{
${ }^{*}$ Corresponding author: Abhishekh Basavarajegowda

Associate Professor, Department of Transfusion Medicine, Jawaharlal Institute of Postgraduate Medical Education and Research(JIPMER), Puducherry, India. 
testing for COVID-19 are summarized in Table.1. The comparison between the molecular-based (RT-PCR) and antigenbased immunoassays are depicted in Table 2.

Serological (Antibody tests) are not recommended for acute infection as it roughly takes two weeks for seroconversion. It only helps in determining the existing immune response to the infection [3].

CT Scan chest should not be used as a standalone test as it lacks specificity, as the similar changes (ground-glass opacities) can be seen in lots of other conditions and infections. It should only be considered in cases suspected to have pulmonary embolism, pneumothorax, or to rule out any other specific pathologies. Chest X-ray is usually inconclusive in the early stages, but as the infection progresses, bilateral multifocal alveolar opacities may be observed, associated with pleural effusion [4].

Other investigations which are of help would be Complete Blood Count CBC, which may show lymphopenia or elevated neutrophil-to-lymphocyte ratio, elevated levels of lactate dehydrogenase, $\mathrm{C}$ reactive protein, creatine kinase (CK MB and CK MM), aspartate aminotransferase and alanine aminotransferase, increased D-dimer levels, and coagulation abnormalities such increase in prothrombin time and the international normalized ratio [5].

Table 1 Testing platforms available

\begin{tabular}{|l|l|}
\hline Molecular testing & RTPCR, CBNAAT \\
\hline Antigen testing & EIA, IFA \\
\hline Antibody testing & Lateral flow assay, ELISA, Chemiluminescence \\
\hline Cell culture & Vero and LLC-MK2 cell lines \\
\hline
\end{tabular}

Table 2 Comparison between molecular (RT-PCR) and Antigen based assays

\begin{tabular}{|l|l|l|}
\hline & RT PCR & Antigen Immuno Assays \\
\hline Sensitivity & 65\% in Nasopharyngeal swab to 93\% in BAL & $80 \%$ compared to RT PCR \\
\hline Specificity & Almost 100\% & $100 \%$ for N protein \\
\hline Logistics & Takes 6-12 hrs., & Rapid, inexpensive \\
\hline Technicality & $\begin{array}{l}\text { Need specialized trained people and infrastructure } \\
\text { of molecular diagnostics }\end{array}$ & $\begin{array}{l}\text { Point of care test, easily } \\
\text { performable in the field with } \\
\text { lesser expertise }\end{array}$ \\
\hline
\end{tabular}

\subsection{Clinical management of COVID 19}

Clinical management of COVID 19 generally involves supportive care for flu-like symptoms in mild and moderate cases with antipyretics and other symptomatic treatment. In severe cases, management is like any other viral sepsis. In severe cases, apart from oxygen therapy, ventilatory care, fluid management, vasopressors, and organ support like renal replacement therapy, no specific pharmacologic treatment is effective with the available evidence. In patients with advanced age associated with comorbidities, terminal illness, and patients with higher clinical frailty score (>6), level of care, including options of non-escalation of care and palliation, should be discussed with family members.

Oxygen therapy can be provided with either nasal prongs (up to 30\% FiO2), oxygen mask (up to 60\% FiO2), or mask with a reservoir (upto90\% FiO2). The aim is to target SpO2 of 90-94\%. Sp02 below 90 may result in tissue hypoxia. Values $>94 \%$, if not monitored, may result in oxygen toxicity apart from wastage of resources. If the Sp02 does not improve with increasing oxygen (15 lts/min), it suggests increased shunt fraction and more severe disease. While the patient is on oxygen, we should continuously monitor the patient work of breathing to assess the patient self-inflicted lung injury (P-SILI)

High flow nasal oxygen (HFNC)/Noninvasive ventilation (NIV): patients with more severe disease, where Sp02 target is not being achieved with oxygen alone may be given a trial of HFNC or NIV. Compared to NIV, HFNC provides better comfort with increased patient compliance and reduced intubation rates.[6] Both these modalities are associated with 
increased aerosolization. So, health care workers providing HFNC and NIV need to have appropriate protection, and these patients should be cared for in designated areas. In patients on HFNC, a surgical mask on the patient will reduce the risk to HCW.

Non-invasive ventilation may be useful in patients with type 2 respiratory failure and cardiogenic pulmonary edema. In patients of COVID 19 with hypoxic respiratory failure (type 1), NIV is associated with increased mortality. NIV trial in such patients should be used only if HFNC is not available. It should be done under close monitoring for work of breathing and early intubation performed in case of deterioration. The interface could be either a face mask or helmet device. It is essential to use only non-vented face masks with exhalation port and (Heat and Moisture Exchanger) HME filter, or two limb circuits with NIV to reduce aerosol spread [7]. The Problem with NIV is that it may delay intubation resulting in deterioration of the patient's condition leading to emergency intubation wherein HCW may be exposed to increased risk. Early experience supports the use of early awake proning in patients with COVID 19 as it increases oxygenation [8][9].

\subsection{Intubation}

Patients with breathlessness who fail the above measures and use accessory muscles of respiration or hemodynamic instability need to be put on invasive mechanical ventilation. Intubation should be performed by the most experienced provider available at the bedside with all aerosol precautions. It is always desirable to have planned elective intubation rather than emergency intubation that can be risky both to the patient and HCWs.

After preoxygenation for 5 minutes, rapid sequence induction should be performed under sedation and paralysis. Induction agents commonly used are etomidate, ketamine, or propofol with opioids fentanyl/morphine. Paralyzing agents used for rapid sequence induction are scoline or rocuronium. A video laryngoscope is recommended as the person intubating the patient can be at a distance while intubating. Start ventilator after reattaching the circuit and inflating the endotracheal tube cuff. Avoid ventilator disconnection all the time to reduce contamination of the atmosphere.

Invasive Mechanical ventilation: Once intubated, patients can be put on either Pressure control mode or volume control mode. As per the ARDS NET protocol, patients are ventilated with a Tidal volume of $6 \mathrm{ml} / \mathrm{kg}$ of ideal body weight (IBW) [10]. Tidal volume can range between $4-8 \mathrm{ml} / \mathrm{kg}$ IBW depending on airway pressures, especially with plateau pressure $<30 \mathrm{cmH} 20$ [11]. It is imperative to limit plateau pressure, as there is enough evidence to suggest an increased risk of ventilator-induced lung injury at higher pressures. PEEP (positive end-expiratory pressure) should be titrated to each patient between $8-15 \mathrm{cmH} 2 \mathrm{O}$. FiO2 or inspired oxygen concentration should be titrated to target $\mathrm{SpO} 2$ of $90-94 \%$.

Tidal volume (TV) can be increased or decreased based on Plateau pressure and driving pressures. Plateau pressure below 30 and driving pressure (Plateau pressure -PEEP) below 15 are desirable to prevent ventilator-induced lung injury [12].

Measuring compliance (TV/Plat-PEEP) will be useful in some cases as it is noted that patients may have two different phenotypes in COVID 19. L- phenotype with low elastane and high compliance ( $>40)$ is seen in the early part of the disease with low lung weight. Prone ventilation may still be useful in this group of patients. H-phenotypes with high elastane and low compliance $(<40)$ are usually seen in more severe disease stages with lung parenchyma flooding. These patients will be responsive to recruitment and high PEEP, apart from prone ventilation.

Prone ventilation: patients with refractory hypoxemia and Pa02/FiO2 ratio of less than 150-200 despite other measures, should be considered for early prone ventilation for more than 16-18 hours per day as it improves both oxygenation and mortality in them [13]. Pressure points, eyes, pupils, and limbs should be continuously monitored. 


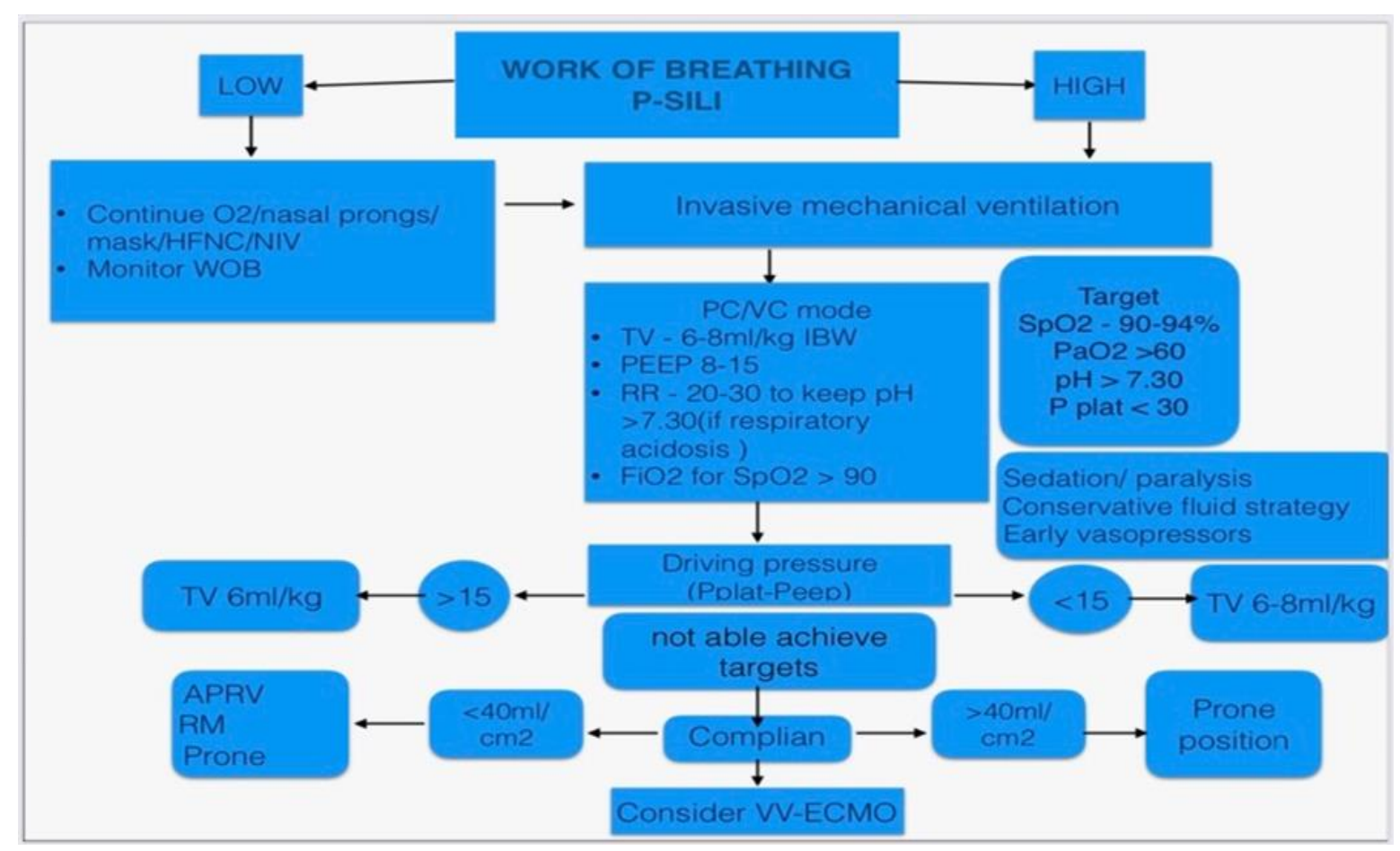

Figure 1 Algorithm for Respiratory management of Covid -19 patients

APRV: Airway pressure release ventilation WOB: Work of breathing TV: Tidal Volume P-SILI: Patient self- inflicted lung injury VV-ECMO: Veno-venous extracorporeal membrane oxygenation.

ECMO: despite all the above supportive measures, if the patient continues to have refractory hypoxemia, Veno-venous ECMO may be contemplated as a last resort, although there is not much evidence to support its use and is associated with increased risks secondary to procedure and anticoagulation.

\subsection{Other supportive therapy}

Anticoagulation: postmortem studies on COVID 19 have shown the presence of micro pulmonary thrombosis that could partially explain hypoxemia with high compliance. Anticoagulation as prophylaxis against Deep Vein Thrombosis (DVT) and Pulmonary embolism (PE) should be started in all patients with no known contraindications. Fibrinolytic agents like defibrotide initiate local fibrinolysis by converting plasminogen to plasmin on the surface of existing thrombi leading to the Improvement of Pa02/FiO2 (P/F) ratio [14].

Fluid management: In patients with COVID 19 and ARDS, they may be in a fluid overload state secondary to cardiogenic pulmonary edema or renal dysfunction. Elderly patients with comorbidities may not tolerate large volume fluid resuscitation. Unless patients have signs suggestive of shock, a conservative fluid strategy should be considered in most patients [15]. We may start low dose vasopressors early to maintain appropriate arterial pressures to aim for a conservative fluid strategy.

Empirical antimicrobials: patients with community-acquired pneumonia should be treated with empiric antibiotics like ceftriaxone, azithromycin, and oseltamivir. Once we get SARS CoV 2 RT PCR report, sputum culture, gram stain, and Influenza viral panel report, these medications can be escalated or de-escalated accordingly.

Role of Corticosteroids: In viral pneumonia (influenza and coronavirus), a Cochrane metanalysis showed an association between steroids and increased mortality. In patients with COVID 19 pneumonia associated with ARDS, a retrospective cohort study showed reduced mortality with steroids [16]. Some recent studies have also demonstrated increased shedding of SARS CoV 2 in patients with Covid 19 on steroids [17]. In conclusion, there is no role in routine steroid use in COVID 19. In selected patients with COVID19 with respiratory failure and ARDS, we should consider steroids in lower doses for a shorter course. 
Role of Lopinavir / Ritonavir: Lopinavir is an antiretroviral protease inhibitor used in HIV. Studies are showing in vitro activity against SARS CoV and in MERS. However, a recent RCT which compared lopinavir/ritonavir to usual care in 199 hospitalized patients with COVID 19 showed no benefit and increased adverse effects like altered LFTs and gastrointestinal symptoms [18]. The ongoing Remap - CAP trial will probably give us more evidence.

Favipiravir: It inhibits the viral RNA synthesis by inhibiting the RNA-dependent RNA polymerases (RdRps). It is known to shorten the recovery time, improve chest image, and has better efficacy than lopinavir-ritonavir [19].

Role of Remdesivir: Multiple trials show in vitro inhibition of SARS CoV 2, SARS CoV, and MERS. Animal studies with MERS showed that Remdesivir was more effective than the control and combination of Lopinavir/Ritonavir with interferon beta [20]. Recent RCT showed that Remdesivir is superior to placebo in shortening time to recovery in adults with COVID 19 [21]. Ongoing trials on remdesivir will throw more light in this regard. National Institutes of Health $(\mathrm{NIH})$ and the Food and Drug Administration (FDA) have recommended it for hospitalized patients with severe COVID19.

Chloroquine/ hydroxychloroquine (HCQ): Both these drugs have shown in vitro effectiveness against SARS CoV 2 [22]. HCQs are more potent than Chloroquine against SARS CoV 2. However, there is insufficient evidence to suggest the routine use of Chloroquine and HCQS. Also, there was an increased incidence of adverse effects associated with HCQs. Monitor corrected QT interval (QTc) in patients on HCQ and azithromycin, and if it is more than 480 msec, consider stopping these medications.

Ivermectin: it is an anti-parasitic drug. Like other drugs mentioned above, in vitro activity against SARS CoV2 induces an approximately 5000-fold reduction in the viral RNA of SARS-CoV-2 at $48 \mathrm{~h}$. A prospective observational casecontrolled study showed a mortality benefit compared to standard care [23]. More research with Randomized Controlled Trials (RCT)s is required to know its usefulness. The dose is $12 \mathrm{mg}$ bd alone or in combination with other therapy for 5 to 7 days is usually given.

Role of Tocilizumab: this is an Interleukin - 6 receptor blocker studied to see if this drug may limit cytokine storm seen in COVID 19. However, the evidence currently insufficient is to suggest its routine use. Tocilizumab is also associated with an increased risk of infections.

Convalescent plasma: Convalescent plasma from patients who have recovered from COVID19 has been used to provide passive immunity against SARS CoV. It is known to reduce fever, decrease virus load, and patients have become negative within 12 days, improves CT im-age, decreases symptoms. However, there is minimal data on its benefit in SARS CoV 2 [24]. There are ongoing trials, and one vast multicentered Randomized Control study is initiated by Indian Council for Medical Research (ICMR) did not show any benefits either concerning the reduction in mortality or in preventing progression to severe COVID-19 [25]. Routine use in COVID 19 is not recommended. Use on Compassionate grounds is allowed as of now in exceptional cases by few states.

Ongoing SOLIDARITY trial in which cases of COVD-19 are randomized to standard care or one of four active treatment arms (remdesivir, chloroquine or HCQs, lopinavir/ritonavir, or lopinavir/ritonavir plus interferon beta-1a) must be followed keenly.

\section{Conclusion}

Although there have been numerous papers in the past few weeks coming in from all over the world about the management of COVID 19, conclusive evidence for any particular modality of treatment is yet to be published. It is pertinent to understand that the successful management of critically ill patients with multi-system involvement requires a concerted holistic approach with multidisciplinary care.

\section{Compliance with ethical standards}

\section{Acknowledgements}

We would like to thank Dr. Nalini. Y.C for helping to edit the manuscript.

\section{Disclosure of conflict of interest}

Nil to disclose. 


\section{References}

[1] Abhishekh B, Channaveerappa B, Srikanth U Shashidhar BG. The Emergence of COVID-19 in India: A LocoRegional, Community Perspective and Its Management. WJRR. 2020; 11: 36-40.

[2] Loeffelholz MJ, Tang Y-W. Laboratory diagnosis of emerging human coronavirus infections-the state of the art. Epub ahead of print. 2020..

[3] Nataraj G, Ingole N. Laboratory Diagnosis of COVID 19 - Perspectives. J Assoc Physicians India. 2020 Jul; 68(7): 11-12.

[4] Cascella M, Rajnik M, Cuomo A, et al. Features, Evaluation, and Treatment of Coronavirus (COVID-19) [Updated 2020 Aug 10]. In: StatPearls [Internet]. Treasure Island (FL): StatPearls Publishing. Jan 2020.

[5] Parasher A COVID-19: Current understanding of its Pathophysiology, Clinical presentation and TreatmentPostgraduate Medical Journal.

[6] Frat J-P, Thille AW, Mercat A, et al. High-Flow Oxygen through Nasal Cannula in Acute Hypoxemic Respiratory Failure. N Engl J Med 2015; 372: 2185-96.

[7] Jan K, Huiling Z. COVID-19: How to safely optimize NIV therapy - Blog | Philips. News center/Global; (cited 28 September 2020).

[8] Elharrar X, Trigui Y, Dols AM, et al. Use of Prone Positioning in Nonintubated Patients with COVID-19 and Hypoxemic Acute Respiratory Failure. JAMA - Journal of the American Medical Association. 2020; 323: $2336-8$.

[9] Prone-Positioning-Protocol-for-Non-Intubated-Patients_Massachussets.

[10] Brower RG, Matthay MA, Morris A, et al. Ventilation with lower tidal volumes as compared with traditional tidal volumes for acute lung injury and the acute respiratory distress syndrome. N Engl J Med. 2000; 342: $1301-8$.

[11] Walkey AJ, Goligher EC, Del Sorbo L, et al. Low tidal volume versus non-volume-limited strategies for patients with acute respiratory distress syndrome: A systematic review and meta-analysis. Annals of the American Thoracic Society. 2017; 14: S271-9.

[12] Amato MBP, Meade MO, Slutsky AS, et al. Driving pressure and survival in the acute respiratory distress syndrome. N Engl J Med. 2014; 372: 747-55.

[13] Guérin C, Reignier J, Richard J-C, et al. Prone Positioning in Severe Acute Respiratory Distress Syndrome. N Engl J Med 2013; 368: 2159-68.

[14] Defibrotide in COVID-19 Pneumonia - Full Text View - ClinicalTrials.gov, (cited 29 September 2020).

[15] Alhazzani W, Møller MH, Arabi YM, et al. Surviving Sepsis Campaign: guidelines on the management of critically ill adults with Coronavirus Disease 2019 (COVID-19). Intensive Care Med. 2020; 46: 854-87.

[16] Lansbury L, Rodrigo C, Leonardi-Bee J, Nguyen-Van-Tam J, Lim WS. Corticosteroids as adjunctive therapy in the treatment of influenza. Cochrane Database Syst Rev. 24 Feb 2019; 2(2): CD010406.

[17] Hui DS. Systemic corticosteroid therapy may delay viral clearance in patients with middle east respiratory syndrome coronavirus infection. American Journal of Respiratory and Critical Care Medicine. 2018; 197: 700-1.

[18] Cao B, Wang Y, Wen D, et al. A Trial of Lopinavir-Ritonavir in Adults Hospitalized with Severe Covid-19. N Engl J Med. 2020; 382: 1787-99.

[19] Cai Q, Yang M, Liu D, et al. Experimental Treatment with Favipiravir for COVID-19: An Open-Label Control Study. Engineering. Epub ahead of print. 2020.

[20] Grein J, Ohmagari N, Shin D, et al. Compassionate use of remdesivir for patients with severe Covid-19. N Engl J Med. 2020; 382: 2327-36.

[21] Beigel JH, Tomashek KM, Dodd LE, et al. Remdesivir for the Treatment of Covid-19 — Preliminary Report. N Engl J Med. Epub ahead of print. 22 May 2020; NEJMoa2007764.

[22] Golden EB, Cho HY, Hofman FM, Louie SG, Schönthal AH, Chen TC. Quinoline-based antimalarial drugs: a novel class of autophagy inhibitors. Neurosurg Focus. Mar 2015; 38(3): E12.

[23] Caly L, Druce JD, Catton MG, et al. The FDA-approved drug ivermectin inhibits the replication of SARS-CoV-2 in vitro. Antiviral Res 2020; 178: 104787. 
[24] Ye M, Fu D, Ren Y, et al. Treatment with convalescent plasma for COVID-19 patients in Wuhan, China. J Med Virol. 2020; 92: 1890-901.

[25] Agarwal A, Mukherjee A, Kumar G, et al. Convalescent plasma in the management of moderate COVID-19 in India: An open-label parallel-arm phase II multicentre randomized controlled trial (PLACID Trial). medRxiv. 2020; 20187252.

\section{Author's short biography}

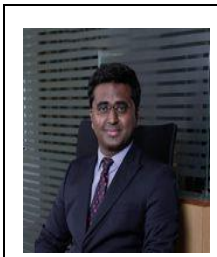

\section{Dr. Shashidhar Binnamangala. MD, FNB, EDIC}

Is a consultant Intensivist in Vikram Hospitals, Bengaluru, a hospital treating many Covid patients' mostly severe cases. He is an excellent clinician with a highly appreciated academic background. His special interest and area of expertise are ARDS and Sepsis.

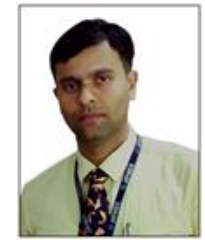

\section{Dr. Abhishekh Basavarajegowda. MD, DNB, MNAMS, PGDCR, Cert Cellular Therapy}

Is an Associate Professor in Transfusion Medicine at Jawaharlal Postgraduate Institute of Medical Education and Research (JIPMER). He was one of the Principal Investigator of the ICMR-PLACID Trial at his site. He has more than 25 publications in various Journals and authored chapters in a couple of books. JIPMER is one of the largest COVID-19 treating hospitals in Southern India.

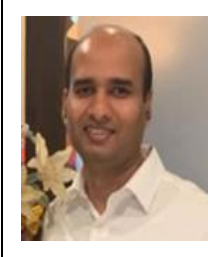

\section{Dr. Srikanth Umakanthan. MD}

Is an academician and Faculty of Medical Sciences, Department of Paraclinical Sciences, Pathology Unit, The University of the West Indies. He is an avid researcher with many publications, including original articles to his credit. He has published a couple of papers on COVID19 in reputed Journals. 